\title{
Calderóns Segismundo und die zweite Natur
}

\author{
Strosetzki, Christoph
}

First published in:

Matzat, Wolfgang; Poppenberg, Gerhard (Hrsg.): Begriff und Darstellung der Natur in der spanischen Literatur der Frühen Neuzeit. München : Fink, 2012, S. 113-122 ISBN: 978-3-7705-5324-2

(C) 2012 Wilhelm Fink Verlag, München 


\section{Calderóns Segismundo und die zweite Natur}

Warum scheitert in Calderóns La vida es sueño Segismundo bei seinem ersten Auftritt im Palast? Warum hat er seine Leidenschaften nicht im Griff und erlaubt sich einen Mord und Zudringlichkeiten, obwohl er doch im Turm eigentlich in Clotaldo einen guten Lehrmeister gehabt hat? Warum mangelt es ihm an den elementarsten Tugenden der Mäßigung und Selbstbeherrschung? Die Fragen sind einfach zu beantworten: Es fehlt ihm die zweite Natur. Im Folgenden soll ausführlich dargelegt werden, welche Bedeutung der zweiten Natur seit der Antike bis zum Siglo de Oro zukommt. Den theoretischen Ausführungen zum Begriff und zu seiner Entwicklung in Philosophie und Theologie soll daher ein größerer Raum zugebilligt werden als den Belegen in der Literatur. Dass es diese jedoch auch gibt, sei schon kurz eingangs gezeigt. Wie wichtig die zweite Natur ist, erklärt Baltasar Gracián im Oráculo manual:

Fueran muchos muy personas si no les faltara un algo, sin el cual nunca llegan al colmo del perfecto ser. Nótase en algunos que pudieran ser mucho si repararan en bien poco. [...] En algunos se desea lo ejecutivo, y en otros lo reportado; todos estos desaires, si se advirtiesen, se podrían suplir con facilidad, que el cuidado puede hacer de la costumbre segunda naturaleza.'

Gleichguiltig also, was fehlt, wird es praktiziert, dann wird nach Gracián aus der Gewohnheit eine zweite Natur. Calderón lässt in seinem Stück El divino Jasón die Figur der Idolatría, Medea, als Musterbeispiel für eine zweite Natur loben, die der ersten Natur weit überlegen ist: «Perderá el sol su belleza, /volará la firme roca, /porque tienes en la boca /segunda naturaleza».2 Und auch Lope de Vega spricht im Zusammenhang mit Gewohnheit und Habitus von der zweiten Natur. ${ }^{3}$

In der heutigen Philosophie wird die Frage gestellt, ob auch Tiere oder unbelebte Gegenstände eine zweite Natur haben können. So kann es dem Hund zur zweiten Natur werden, sich hinzusetzen, wenn er den Befehl «Platz!» hört. John Mc Dowell kommentiert: «Apart from how it originates, the second na-

I Baltasar Gracián: El Héroe. Oráculo manual y arte de prudencia, hg. v. Antonio Bernat Vistarini/Abraham Madroñal, Madrid: Castalia, 2003, S. 301.

2 Pedro Calderón de la Barca: El divino Jasón, hg. v. Ignacio Arellano/Ángel L. Cilveti, Pamplona: Universidad de Navarra, 1992, S. 171.

3 Vgl. Lope de Vega: La Arcadia, hg. v. Edwin S. Murby, Madrid: Castalia, 1975, S. 382: «Aquí veréis el efeto que hace la ciencia, cuyo ejercicio honesto priva todo pensamiento ocioso, sacando el alma del cautiverio de la vil costumbre y rompiendo el hábito estrecho convertido en la misma vida, como segunda naturaleza. Veréis cómo se puede seguir la virtud, sin que espanten sus ásperos principios, y cómo no hay dificultad en ella que, esforzando la voluntad, no se acabe con la paciencia y consiga con la perseverancia.» 
ture of dogs is just like their first nature». ${ }^{4}$ Gold kann in seiner ersten Natur an Steinen heften oder sich als Edelmetallklumpen im Fluss befinden, während es eine zweite Natur erhält, wenn es zu Goldbarren oder Goldringen verarbeitet wird. Wenn Kinder inmitten sozial und historisch gebildeter Artefakte und Traditionen aufwachsen, profitieren sie - anders als Segismundo - von dem akkumulierten Wissen und den sozialen Fertigkeiten ihrer Gruppe. Wenn die biologische nicht durch eine kulturelle Vererbung ergänzt wird, erscheinen dem Philosophen Michael Tomasello Bildung und Reifung unmöglich. ${ }^{5}$

In der Antike wurde der ursprüngliche Zustand der Seele als tabula rasa bezeichnet, was bedeutet, dass die Eigenschaften, Inhalte und Charakteristika erst im Laufe der Zeit hinzukommen. ${ }^{6}$ Im Griechischen kommen Physis (Natur) und Hexis (Habe, Haltung) zusammen, wenn Platon hinsichtlich der Erziehung der Wächter im Staat konstatiert, dass «Nachahmungen, die man von Jugend an beständig betreibt, zur Gewohnheit und zur anderen Natur werden, ob das nun den Leib oder die Stimme oder die Denkart betrifft». ${ }^{7}$ Dabei reicht die bloße Wiederholung nach Aristoteles nicht aus. Hinzukommen muss ein innerliches Aneignen, eine Habe (Hexis), ein sich Verhalten in bestimmter Art, bei dem man bleibt. ${ }^{8}$ Wenn Hexis die Habe ist, die auch fehlen kann, dann stellt sich ontologisch für Aristoteles die Frage, ob es neben der Natur noch eine andere Natur geben kann und ob es bei der Hexis möglich ist, dass ein Substrat etwas haben und zugleich entbehren kann, obwohl es dies als ganze Natur besitzen müsste. ${ }^{9}$ Aristoteles sei etwas ausführlicher herangezogen, da er in der spanischen Spätscholastik des Siglo de Oro zentraler Referenzphilosoph war. Während die Verstandestugenden seiner Meinung nach durch bloße Belehrung angeeignet werden könnten, sei dies bei den sittlichen nicht der Fall, da diese nur durch Gewöhnung erworben werden. Während beim Sehen dem Besitz der Gebrauch gefolgt ist, sei es bei den Tugenden umgekehrt:

Die Tugenden dagegen erlangen wir nach vorausgegangener Tätigkeit, wie dies auch bei den Künsten der Fall ist. Denn was wir tun müssen, nachdem wir es gelernt haben, das lernen wir, indem wir es tun. So wird man durch Bauen ein Baumeister und durch Zitherspielen ein Zitherspieler. Ebenso werden wir aber

4 Vgl. John McDowell: «Responses», in: Marcus Willaschek/John McDowell (Hg.): Reason and Nature. Lecture and Colloquium in Münster 1999, Münster: Lit Verlag, 2000, S. 98.

5 Michael Tomasello: Die kulturelle Entwicklung des menschlichen Denkens, Frankfurt a. M.: Suhrkamp, 2002, S. 24.

6 Platon: Theaitetos (191c), in: ders.: Sämtliche Werke, Bd. 4, übers. v. Friedrich Schleiermacher, hg. v. Walter F. Otto u.a., Reinbek: Rowohlt, 1967, S. 159; Aristoteles: Über die Seele, übers. v. Willy Theiler, hg. v. Horst Seidl, Hamburg: Felix Meiner Verlag, 1995, S. 170-171.

7 Platon: Staat (III, 395d), gr./dt., übers. v. Rudolf Rufener, hg. v. Thomas A. Szlezák, Düsseldorf: Artemis \& Winkler, 2002, S. 219.

8 Aristoteles: Rhetorik (I, 11, 1370 a u. I, 10, 1369 b6), übers. v. Christof Rapp, hg. v. Hellmut Flashar, Berlin: Akademie, 2002, S. 53f.

9 Aristoteles: Metaphysik (V, 19-20, 1022 b 1-J4), übers. v. Hermann Bonitz, hg. v. Ursula Wolf, Reinbek: Rowohlt, ${ }^{2} 1999$, S. 155-156; Aristoteles, Kategorien (10, 12 a 29), übers. v. Klaus Oehler, hg. v. Klaus Flashar, Darmstadt: WBG, 1984. 
auch durch gerechtes Handeln gerecht, durch [Einhaltung] der Mäßigkeit mäßig, durch Werke des Starkmuts starkmütig. ${ }^{10}$

Nur wer es gewohnt ist, Gefahren zu verachten, gewinnt die Tugend der Tapferkeit. Wer tapfer ist, wird dann leichter Gefahren bestehen können. Dass Tugend nicht durch Belehrung, sondern nur durch Praxis entsteht, sei an einem weiteren aristotelischen Zitat veranschaulicht:

Für die Tugend aber bedeutet das Wissen wenig oder nichts, das andere dagegen, was nur durch fortgesetzte Übung der Gerechtigkeit und Mäßigkeit erworben wird, bedeutet nicht wenig, sondern alles. [...] Es ist also richtig gesprochen, dass man durch Handlungen der Gerechtigkeit ein gerechter und durch Handlungen der Mäßigkeit ein mäßiger Mann wird. Niemand aber, der sie nicht verrichtet, ist auch nur auf dem Weg, tugendhaft zu werden. ${ }^{11}$

Es kommt also darauf an, einen Habitus zu entwickeln, der es z.B. ermöglicht, sich hinsichtlich der Affekte richtig zu verhalten, das heißt zum Beispiel in Bezug auf den Zorn die rechte Mitte zu finden und ihn nicht exzessiv werden zu lassen. Daher bezeichnet Aristoteles die Tugend des Menschen als Habitus, der es ihm erlaubt, gut zu sein und seine Werke gut zu verrichten. Eben dieser Habitus ist bei Aristoteles als zweite Natur zu verstehen.

Auch im christlichen Kontext ist die Lehre von der zweiten Natur bekannt, nach der einer natürlichen Anlage eine erworbene Anlage hinzugefügt werden muss, deren Charakteristikum ist, dass sie auch fehlen könnte. Zur Ausbildung solcher Anlagen heißt es im Hebräerbrief des Neuen Testaments, dass demjenigen, der nach dem Geiste lebt, das zugeführt werden soll, was dem Geiste dient, so dass denen die starke Speise gebührt, deren Sinne durch Gewohnheit geübt in der Lage sind, Gutes und Böses zu unterscheiden (Hebr 5). Mit Blick auf die Zehn Gebote des Alten Testaments formuliert Philon noch pointierter, dass Gewohnheit, wenn sie andauert, stärker sein kann als Natur. Dass der Begriff der «zweiten Natur〉 zur Bezeichnung der Gewohnheit im Kontext der patristischen Philosophie bereits eingebürgert war, zeigt Macrobius, wenn er von «Consuetudo, quam secundam naturam pronuntiavit usus» spricht. ${ }^{12}$

Eine etwas andere Perspektive bringt Augustinus ein, wenn er mit der zweiten Natur die Natur des nach dem Sündenfall aus dem Paradies vertriebenen Menschen meint und die Natur des Menschen im Paradies zur ersten Natur macht. Bei Augustinus und bei Thomas von Aquin wird die Macht der Gewohnheit auf die Notwendigkeit der Gnade bezogen. Will der Mensch die Gewohnheit des Lebens nach dem Fleische durch die des Lebens nach dem Geiste ersetzen, bedarf er nach Augustinus übernatürlicher Hilfe, ohne die ein

10 Aristoteles: Nikomachische Ethik, in: ders.: Philosophische Schriften, Bd. 2, übers. v. Eugen Rolfes, hg. v. Günther Bien, Hamburg: Meiner, 1995, S. 26-27.

11 Ebenda, S. 32.

12 Ambrosius Theodosius Macrobius: Samınalia $(7,9,7)$, hg. v. Jacob Willis, Leipzig: Teubner, 1963, S. 429. 
solcher Habitus nicht zu erreichen wäre. ${ }^{13}$ Man kann daher zwischen einem habitus acquisitus, der durch intensive Betätigung erreicht wird, und einem habitus infusus unterscheiden, der durch übernatürliche göttliche Einwirkung geschieht. ${ }^{14}$ Der spanische Humanist Juan de Pineda zieht die «hábitos adquiridos» den «hábitos infusos» vor, wenn er von der Tugend spricht:

que se hace y torna como connatural al hombre y le conserva en el bien; porque, aunque los hábitos no confirmen al operante en sus actos, y aún los hábitos infusos no inclinen, según Escoto, a lo menos los adquiridos por costumbre, de los cuales se dice ser como segunda naturaleza, inclinan ihucho a tales obras, cuales fueron aquellas que los engendraron; y ansí permanecen los virtuosos constantes en el servientes de la Iglesia. ${ }^{15}$

Ein spezielles Problem ergibt sich in diesem Zusammenhang für Huarte, wenn er nach der Möglichkeit einer "sabiduría infusa» ${ }^{16}$ bei Christus fragt und dabei davon ausgeht, dass die göttliche Weisheit beim Gottessohn Resultat einer ersten und nicht einer zweiten Natur sein könne. Verlassen wir speziellere theologische Fragen und kehren zu den allgemeinen zurück.

In seinem Traktat über die Gewohnheiten und Tugenden erklärt Thomas von Aquin, dass Gewohnheiten (hábitos, habitus) wie die Wärme, die das Feuer erzeugt, durch Handlungen erzeugt werden, also potentia wird durch actıs erzeugt. "Porque todo lo que es pasivo y movido por otro, recibe una disposición por la actividad del agente. Por lo cual, la repetición de actos llega a formar en la potencia - en cuanto que es pasiva y movida - una cierta cualidad llamada hábito.» ${ }^{17} \mathrm{Im}$ darauf folgenden Artikel insistiert er: «Acabamos de decir que el hábito se engendra por actos, en cuanto que la potencia pasiva es actuada por un principio activo. ${ }^{18}$ Es sei wie beim Feuer, das den Gegenstand nicht sofort verbrennt, sondern nach und nach die sich ihm entziehenden Dispositionen überwindet und so schließlich das Ganze besiegt und ihm seine Form aufdrängt. Der Habitus ist nach Thomas vom Wesen her eine Perfektion, die allerdings quantitativ vermehrt oder vermindert werden kann. Wie nicht jede einzelne Nahrung zum Wachsen und Erstarken eines Tieres führe und nicht jeder einzelne Tropfen den Stein aushöhle, so ist es auch mit den Handlungen: «repetidos los actos con igual o superior intensidad,

13 Aurelius Augustinus: Confessiones/Bekenntnisse (VIII, 5), lat./dt., übers. v. Wilhelm Thimme, Düsseldorf: Artemis \& Winkler, 2004.

14 Tomás de Aquino: Suma teológica, hg. v. Francisco Santiago Ramirez, Madrid: BAE, 1954, S. 91-93.

15 Juan de Pineda: Diálogos familiares de agricultura cristiana, hg. v. P. Juan Meseguer Fernández, Madrid: Atlas, 1963, S. 285-286.

16 Huarte de San Juan: Examen de ingenios para las ciencias, hg. v. Guillermo Serés, Madrid: Cátedra, 1989, S. 681.

17 Tomás de Aquino: Suma teológica (Anm. 14), S. 88. («Nam omne quod patitur et movetur ab alio, disponitur per actum agentis: unde ex multiplicatis actibus generatur quaedam qualitas in potentia passiva et mota, quae nominalur habitus.»)

18 Ebenda, S. 89. («Respondeo dicendum quod, sicut iam dictum est, habitus per aclum generatur inquantum potentia passiva movetur ab aliquo principio activo.») 
se produce el aumento. En este mismo sentido, la repetición de actos aumenta los hábitos.»' ${ }^{19}$ Im Gegenschluss führt ein Schwinden der Intensität der Handlungen zur Schwächung des Habitus. Wenn nun allerdings Thomas zwischen guten und schlechten Handlungen unterscheiden will, muss er doch auf die menschliche Natur zurïckgreifen:

por razón de la conformidad o disconformidad con la naturaleza. [...] Se dice bueno o malo, según que disponga para un acto conveniente o no con la naturaleza del que obra; así, los actos de las virtudes son convenientes con la naturaleza humana; y los hábitos malos disponen a actos no convenientes con la misma. $^{20}$

Als Kriterium der Beurteilung des jeweiligen Habitus wird also die menschliche Natur angeführt, die hier im aristotelischen Sinn als Form- und Zielursache zugleich für Wesen und Perfektion des Menschen steht. Dass beim Menschen vor allem individuelle Praxis erforderlich ist, um «sólidas virtudes»" zu erhalten, unterstreicht im Sinne von Thomas Ignatius von Loyola: «Porque esforzándose de su parte en vencer a sí mismo y deshacer el amor propio, quitan con él las raíces de todas las pasiones y molestias; y también con alcanzar los hábitos virtuosos, vienen como naturalmente a obrar conforme a ellos, fácil y alegremente» (S. 723). In Loyolas Ejercicios espirituales wird in einer Regel zwischen einer Disposition zum Negativen und einer Disposition zum Positiven unterschieden:

porque entonces propio es del mal spíritu morder, tristar y poner impedimentos inquietando con falsas razones, para que no pase adelante; y propio del bueno dar ánimo y fuerzas, consolaciones, lágrimas, inspiraciones y quietud, facilitando y quitando todos impedimentos, para que el bien obrar proceda adelante (S. 227).

An anderer Stelle variiert Ignatius den Gedanken und unterscheidet zwischen den verschiedenen Auswirkungen der Dispositionen: «mucho mal a los que tienen la voluntad depravada y hábitos malos; mucho bien a los que tienen la voluntad toda a Dios N. S. aplicada y en buenos hábitos acostumbrada» (S. 665). Es zeigt sich also, dass Calderón nicht nur bei den Philosophen, sondern auch bei den Theologen mit den Theorien zur zweiten Natur vertraut werden konnte.

In weiterführenden und auch späteren philosophischen Diskussionen ergaben sich aus der Vorstellung von der Seele als tabula rasa und der Aneignung

19 Ebenda, S. 117. («[...] sed, multiplicato alimento, tandem fit augmentum. Ita etiam, multiplicatis actibus, crescit habitus.»)

20) Ebenda, S. 146. («Uno modo, secundum convenientiam ad naturam, vel etiam secundum disconvenientiam ab ipsa. [...] nam habitus bonus dicitur qui disponit ad actum convenientem naturae agentis; habitus autem malus dicitur qui disponit ad actum non convenientem naturae. Sicut aclus virtutum naturae humanae conveniunt, eo quod sunt secundum rationem: actus vero vitiorum, cum sint contra rationem, a natura humana discordant.»)

21 San Ignacio de Loyola: Obras completas, Madrid: BAC, 1952, S. 612; als «virtudes sólidas» werden "paciencia, humildad, obediencia, abnegación de la voluntad propia, caridad» verstanden (S. 857). 
der ethischen Tugenden durch Nachahmung und Gewöhnung zwei Fragen. Welche Bedeutung haben die Sitten für die Herausbildung der zweiten Natur? Gibt es überhaupt eine erste Natur? Wenn es von Natur aus nichts gibt, was aus sich heraus Gültigkeit hat, dann sind es für den Skeptiker Montaigne die in der Gesellschaft verbreiteten guten Sitten, die nachzuahmen sind und die zur persönlichen Gewohnheit werden sollen. Mit Plinius konstatiert er: «usus efficacissimus rerum omnium magister. ${ }^{22}$ Bei Hegel später werden die Gewohnheiten der Sitten als zweite Natur bezeichnet, als eine Welt vorhandenen Geistes, in der die Subjekte wirklich leben. Während die Natứ für das Bewusstsein nach Hegel etwas ihm Fremdes ist, ist die zweite Natur eine höhere Natur: «Für das Subjekt haben die sittliche Substanz, ihre Gesetze und Gewalten [...] eine absolute, unendlich festere Autorität und Macht als das Seyn der Natur.. ${ }^{23}$

Dass die zweite Natur mächtiger ist als die erste, behauptete aber auch schon vor dem deutschen Idealismus Pascal lapidar mit dem Satz: «La coutume est une seconde nature, qui détruit la première.» Daraus ergibt sich für ihn nicht nur die Dominanz der zweiten über die erste Natur, sondern sogar die Frage, ob überhaupt die erste von der zweiten Natur zu unterscheiden ist bzw. ob es nicht vielleicht nur eine so genannte «zweite Natur» gibt: «J'ai grand peur que cette nature ne soit elle-même qu'une première coutume, comme la coutume est une seconde nature». ${ }^{24}$

Hier kann man unterscheiden zwischen einer subjektiven Gewohnheit, die z.B. in Funktionsstärkung bzw. Abstumpfung, Stärkung des Geistigen mit Schwächung des Körperlichen besteht, und auf der anderen Seite kollektiven Gewohnheiten, die als Bräuche Zwangs- und Normierungscharakter haben. Der subjektiven Aneignung steht die objektive Üblichkeit gegenüber. Wenn wie bei Hobbes die Gegenstände der Welt entweder Körper oder Bewegungen sind, dann bewirkt die Gewohnheit das Entstehen einer Bewegung, das leichte Hinführen eines bewegten Körpers auf einen bestimmten vorgezeichneten Weg, wobei Aversionen überwunden und Neigungen gefördert werden. ${ }^{25}$ Bei der subjektiven Gewohnheit kommen der Übung, der Wiederholung und dem Gedächtnis besondere Bedeutung zu.

Kommen wir zurück zur Diskussion in Spanien. Durch Wiederholung derselben Handlungen entstehen Gewöhnungsprozesse und mit ihnen Prädispositionen, die die Verläufe schließlich wie quasi-instinktiv und gleichsam automatisch ohne jeweiliges Eingreifen vernünftigen Entscheidens ermöglichen.

22 Plinius, Natıralis Historia, 26,6; vgl. Michel de Montaigne: Les Essais (1,23), hg. v. Pielte Villey/V. L. Saulnier, Paris: Quadrige, 2004.

23 Georg Wilhelm Friedrich Hegel: Grundlinien der Philosophie des Rechts oder Naturrecht und Staatswissenschaft im Grundrisse ( $\S 146)$, hg. v. Hermann Glockner, Stuttgart-Bad Cannstatt: Frommann, 1964.

24 Blaise Pascal: Pensées, hg. v. Philippe Sellier, Paris: Éditions Classiques Garnier, 2010, S. 219 (Fr.159).

25 Thomas Hobbes: Vom Körper (22, 20), übers. v. Max Frischeisen-Köhler, Hamburg: Felix Meiner, ${ }^{31967, ~ S . ~} 132$. 
Bei der memoria unterscheidet der spanische Humanist Vives memoria, recuerdo und reminiscencia - eine Unterscheidung, auf die wir nicht näher eingehen wollen. In der Tat ist es ein Verdienst des Gedächtnisses, dass sich ein Habitus und eine zweite Natur aufbauen, «porque la memoria le ayuda, no sólo para recibir fácilmente lo que se desea, sino para reproducirlo pronto y con fidelidad cuando sea necesario». ${ }^{26}$

Des Weiteren erklärt Vives, Aristoteles und Thomas folgend, dass die Handlungen durch Fähigkeiten erzeugt werden. Die einen, wie z.B. das Sehen und Hören, sind beim Neugeborenen von vornherein vorhanden und benötigen keine Übung, um gut zu gelingen, «sino que tienen plena madurez y vigor, como amaestrados por virtud de la naturaleza misma». ${ }^{27}$ Die anderen komplexeren Handlungen aber benötigen nach Vives Praxis, Übung und Gewöhnung, «en la cual se reúnen facilidad para obrar y propensión, y viene del vocablo griego 〈Hexis», que corresponde al habitus〉, o sea la inclinación a realizar actos semejantes a aquellos de los cuales se formó.» Wie der Habitus Handlungen nahelegen bzw. festlegen kann, so kann er dies auch bei Leidenschaften tun. Gerade dann ist die Gewohnheit gefordert, wenn es darum geht, mit Leichtigkeit «formas absurdas, repugnantes, y que causan miedo, o sonidos estupendos y horribles, sabores ácidos y amargos» zu ertragen. «De esa costumbre nacen la aptitud y facilidad en el artífice y la correspondiente adecuación en el instrumento.» Als Beispiel führt Vives den Maler an, dessen Hand den Pinsel nur durch Gewohnheit und praktische Einübung sicher führt. Erst die Gewohnheit führt zur sicheren Disposition beim Malen. «Cuando un hábito se arraiga por el uso continuo adquiere casi fuerza de naturaleza, invitándonos a obrar de igual modo sin dificultad y hasta con gusto.» Gewohnheit macht die alltäglichen Dinge angenehmer, und auch das Unangenehme ist durch Gewohnheit erträglicher. So hält es Vives für besonders wichtig, «el mejor método de vida» zu wählen, «porque con la costumbre se hará llevadero, y según Platón es de gran importancia, el modo cómo se ha formado y acostumbrado cada uno desde la niñez». Damit unterstreicht Vives die Bedeutung der Einübung eines Habitus für das richtige Handeln. Vives bezeichnet den Habitus als Grundbaustein der Seele, «a fin de suministrarla mediante el uso y el ejercicio cuanto debemos hacer que sea recto y conveniente». Wäre es nämlich nicht so, dass durch Kontinuität in der Zeit die Fähigkeit, in derselben Richtung zu handeln, bestärkt würde, dann würde man immer wieder am Anfang stehen und es wäre nicht möglich, etwas Ausgezeichnetes hervorzubringen. Es hat sich also gezeigt, dass der Begriff der zweiten Natur, wie er in der Antike konzipiert wurde, nicht nur in der Literatur, sondern auch in der Philosophie und Theologie des spanischen Siglo de Oro präsent ist. Mateo Alemán stellt Bedeutung und Komplexität der zweiten Natur im zweiten Teil des Guzmán de Alfarache besonders ausführlich vor, da

${ }^{26}$ Luis Vives: Tratado del alma, hg. v. José Ontañón, Buenos Aires: Espasa Calpe, ${ }^{2}$ 1945, S. 60.

27 Ebenda, S. 112-113 (gilt auch für die folgenden Zitate). 
es für seinen Pícaro nicht unerheblich ist, mit welchen Handlungen er sich welche Gewohnheiten angeeignet hat. Es stellt sich für ihn die Frage, ob er die Möglichkeit hat, sich gegen die Macht der Zeit und der Gewohnheit zu wehren. Denn mächtig ist die Gewohnheit nach Mateo Alemán zum Guten wie zum Schlechten hin:

Quita y pone leyes, fortaleciendo las unas y rompiendo las otras; prohíbe y establece, como poderoso príncipe, y consecutivamente a la parte que se acuesta, lleva tras de sí el edificio, tanto en el seguir los vicios, cuanto en ejercitar virtudes. [...] No hay fuerzas que la venzan y tiene dominto sobre todo caso. Algunos la llamaron segunda naturaleza, empero por experiencia nos muestra que aún tiene mayor poder, pues la corrompe y destruye con grandísima facilidad. $^{28}$

Die Gewohnheit ist so stark, dass sie das Geschmacksempfinden verändern kann: Wenn Bitteres gut schmeckt, dann ist es nur der Gewohnheit zu verdanken, die es versüßt. Nur die Gewohnheit, die zur zweiten Natur geworden ist, macht es nach Mateo Alemán dem Schäfer erträglich, in verlassenen Feldern, tiefen Tälern oder hohen Gipfeln und Gebirgen harte Winter, Unwetter, Regen und Stiirme oder im Sommer die unerbittliche Sonne und die erhitzten Steine und Metalle zu ertragen. Die Gewohnheit sei stark wie die Sonne. Während letztere mit ihrem Gold Sein und Leben spende, könne die Gewohnheit im Stillen stärken, schaffen, zerstören und «con las continuas gotas cavar las duras piedras» (S. 579). Stärker als die Gewohnheit sei nur die Zeit, von der sie abhängig sei. In Verbindung mit der Zeit habe sie so viel Gewalt, «que hace domesticarse las fieras más fieras y ponzoñosas, refrenando sus furias y mitigando sus venenos» (S. 578). Dies alles, sagt der Protagonist Mateo Alemáns, habe er durch seine eigenen Erlebnisse bestätigen können: «Todo lo dicho se verificó bien de mí, en proprios términos y casos» (S. 579). Die Handlungen des Pícaro in der Gesellschaft haben also seine Gewohnheiten und seinen wenig moralischen Habitus ausgeprägt. Anders ist es mit dem bestellt, der keine Gelegenheit hatte, in der Gesellschaft Handlungen einzuiiben und damit Gewohnheiten auszubilden. Er entspricht Mateo Alemáns Bild vom wilden Tier, dessen Raserei und Gewalttätigkeit noch nicht durch gute Gewohnheiten domestiziert ist.

Hier kann man an Segismundo denken, wenn er zum ersten Mal nach seinem Aufenthalt im Turm mit dem Palast konfrontiert wird. Er hatte nicht die Gelegenheit, durch Handeln in der Gesellschaft seine sittlichen Gewohnheiten zum Guten oder zum Schlechten auszubilden. Hat sich Mateo Alemáns Pícaro durch seine Handlungen eine schlechte Gewohnheit zur zweiten Natur gemacht, so konnte Segismundo keine Gewohnheit durch Handeln ausbilden, weshalb er seiner Herausforderung auch nicht gewachsen ist. Denn während im Bereich der Erkenntnis die Theorie ausreicht, ist die Ausbildung sittlicher

28 Mateo Alemán: Guzmán de Alfarache, hg. v. Benito Brancaforte, Madrid: Akal, 1996, S. 578. Auch die weiteren Zitate entstammen dieser Ausgabe. 
Tugenden von der praktischen Ausübung abhängig. Wie fremd die Welt für den im Turm eingesperrten Segismundo ist, wird gleich am Anfang bei seiner Begegnung mit Rosaura deutlich, wenn er sie fragt, wer sie ist: «tan poco del mundo sé, / que cuna y sepulcro fue / esta torre para mí». ${ }^{29}$ Wenn er seinen Aufenthaltsort als «rústico desierto» bezeichnet, ist das schon fast eine Anspielung auf die ihm korrespondierende seelische tabula rasa. Und wenn er eingesteht, dass er das, was er ïber Himmel und Erde weiß, nur von einer Person, nämlich Clotaldo, durch Erziehung erfahren hat, ist es natürlich, dass er sich als «un hombre de las fieras y una fiera de los hombres» (S. 41) bezeichnet. Die Gewohnheit des gesellschaftlichen Umgangs ist es, die ihm fehlt. Wie man einen Staat lenkt, hat er nicht bei Menschen, sondern bei Tieren in Erfahrung gebracht: «la política he estudiado, / de los brutos enseñado» (S. 41). Die letras humanas, meint Clotaldo, habe Segismundo gelernt «de los montes y los cielos, / en cuya divina escuela / la retórica aprendió / de las aves y las fieras» (S. 73).

Dass Segismundo sich dann im Palast, wo alles anders als gewohnt ist, völlig überfordert sieht, verdeutlichen seine ersten Ausrufe: «Válgame el cielo, qué veo! / Válgame el cielo, qué miro! / Con poco espanto lo admiro, con mucha duda lo creo. / Yo en palacios suntuosos? / Yo entre telas y brocados?» (S. 79). Dass Segismundo Einschränkungen und Rücksichtnahmen nicht gewohnt ist und Hindernisse rüicksichtslos beseitigt, zeigt sein programmatischer Satz: «No me estorbe nadie, / que es vana diligencia, y, / si os ponéis delante vos, / que os eche por la ventana» (S. 82). Es bringt ihm seitens Clotaldo den Vorwurf der superbia ein. Immer wieder wird hervorgehoben, dass es Tiere sind, unter denen Segismundo aufgewachsen ist. Segismundo wirft Basilio vor, er habe ihn verstoßen und «como a una fiera me cría, / y como un monstruo me trata» (S. 89). Später wird er konstatieren: "y sé que soy: / un compuesto de hombre y fiera» (S. 91). Rosaura wird Segismundo die menschliche Natur absprechen: «Mas qué ha de hacer un hombre / que de humano no tiene más que el nombre, / atrevido, inhumano, / cruel, soberbio, bárbaro y tirano, / nacido entre las fieras?» (S. 96). Seine darauf folgenden Handgreiflichkeiten werden mit enojo, rigor, rabia und cólera bezeichnet, Charakteristika also, die traditionell nicht einer wohl ausgebildeten zweiten Natur des Menschen, sondern eher der tierischen Natur zugeschrieben werden.

Wenn wir einmal davon absehen, dass nach neuerer philosophischer Forschung auch Hunde und Edelmetalle eine zweite Natur haben können, ist festzuhalten, dass die zweite Natur im Verständnis des Siglo de Oro dem Menschen vorbehalten ist. Hier greift man besonders auf Aristoteles zurück, der das Vorhandensein von Tugenden ohne vorausgegangene Tätigkeit für unmöglich hält und das Wissen um Tugenden gering schätzt, weil Tugenden nur durch fortgesetzte Übung erworben werden. Voraussetzung für sie ist, dass

29 Pedro Calderón de la Barca: La vida es sueño, hg. v. Domingo Ynduráin, Madrid: Alianza, 2002, S. 40-41. 
sich ein Habitus einstellt, der zur zweiten Natur wird. Ohne den Habitus ist der Ausgang von Handlungen als Reaktionen auf Situationen zufällig und unsicher. Deshalb spricht Ignatius von «sólidas virtudes» und die theologische Diskussion bestätigt den philosophischen Ansatz und bereichert ihn, indem sie die göttliche Gnade ins Spiel bringt. Beide Ansätze kulminieren in der von Pascal wie von Hegel gestellten Frage, ob es überhaupt eine erste Natur gibt bzw. ob die zweite Natur nicht über die erste dominiert, ja diese vielleicht sogar zerstört. Vives war es, der sehr plastisch in Anlehnung an Aristoteles darstellte, wie aus der seit der Kindheit ausgeübten Praxis der Hábitus und aus diesem die auf das richtige Handeln ausgerichtete Persönlichkeit entsteht.

Dass ohne Ausbildung eines Habitus das harte Leben eines Schäfers nicht auszuhalten wäre, erläuterte Mateo Alemán. Dass Entsprechendes für das Leben am Hof bzw. überhaupt in menschlicher Gesellschaft gilt, dafür war Segismundo ein Beispiel. Ihm war es nicht möglich, unter den Tieren einen geeigneten Habitus, eine passende zweite Natur auszubilden. Und auch die Lehren von Clotaldo waren nur theoretisch. Praxis, Übung und Gewöhnung waren ihm nicht vergönnt. So lässt sich resümierend konstatieren, dass Segismundo bei seinem ersten Auftritt im Palast weder wegen eines schlechten Charakters noch wegen der Gestirne und der aus ihnen abgeleiteten Voraussage scheiterte, sondern weil es ihm an der Praxis fehlte, mit der er seine Tugenden hätte ausbilden können. Ohne die Ausbildung eines Habitus fehlt ihm die notwendige zweite Natur. So handelt er gemäß der ersten Natur, deren Praxis er unter den wilden Tieren kennen gelernt hat, da es bei ihnen keine zweite Natur gibt. So gesehen ist Calderóns Stück ein Plädoyer für höfische Kultur und Geselligkeit und ein Beleg für die schlimmen Folgen, die sich aus einem Entfernen oder Entferntwerden vom Hof und von der Gesellschaft ergeben. 